\title{
Grouping effects in flash-induced perceptual fading
}

\author{
Mark L T Vergeer, Rob van Lier \\ Nijmegen Institute for Cognition and Information ( $\mathrm{NICl}$ ), Radboud University Nijmegen, PO Box 9104, \\ 6500 HE Nijmegen, The Netherlands; e-mail: m.vergeer@nici.ru.nl \\ Received 5 April 2006, in revised form 24 July 2006; published online 11 May 2007
}

\begin{abstract}
We show that the flash-induced fading effect can be influenced by grouping based on colour and shape similarity. In flash-induced fading, peripherally presented elements perceptually disappear after a flash has been presented around or next to the element(s) (Kanai and Kamitani, 2003 Journal of Cognitive Neuroscience 15 664-672). In the present study, two elements (which could either be similar or different in both colour and shape) were presented at an eccentricity of $2.6^{\circ}$ from a fixation cross. After $2 \mathrm{~s}$, a short flash $(40 \mathrm{~ms})$ was presented around one of the elements. The two elements remained on the screen for $2.5 \mathrm{~s}$ after the flash. We found a higher percentage of mutual perceptual disappearance when the two elements were similar in colour or in shape. These results converge with other findings showing that perceptual fading phenomena are influenced by effects of perceptual grouping. This indicates that, besides low-level adaptation, more global processes play a part in perceptual fading as well and should thus be taken into account in giving a full explanation of perceptual fading in general.
\end{abstract}

\section{Introduction}

Various studies on visual functioning have shown that, during perception, visual information can sometimes 'escape' from awareness. As early as 1804 Troxler reported that fixating on a spatial location, while attending to an element in the peripheral visual field, could lead to a temporary spontaneous perceptual disappearance of the peripherally presented element. In a related perceptual-disappearance phenomenonmotion-induced blindness (MIB) - one or more stationary elements, presented on a background containing coherently moving elements (eg dots or crosses), disappear from awareness for several seconds (Bonneh et al 2001). A phenomenon which can also be related to the traditional Troxler effect and which, like MIB, is caused by a visual transient, is the so-called flash-induced perceptual fading phenomenon (Kanai and Kamitani 2003), in which a ring is flashed around a stationary peripherally presented disc for about $40 \mathrm{~ms}$. The disc is present during the whole trial, but the flash triggers its temporary perceptual disappearance. This effect differs from MIB; whereas the 'distracting' or 'fading-triggering' information is continuously present in MIB, this information is only presented for a short period in flash-induced fading.

Although local adaptation to luminance edges, which can be considered to occur early in the visual process, is commonly seen as the main cause of Troxler fading (eg Ramachandran and Gregory 1991), an interesting question is to what extent other, more global (possibly higher-level) processes also influence which information will disappear in perceptual fading. Already, some studies have addressed the question of the extent to which different peripheral fading phenomena are influenced by perceptual grouping. In their MIB study, Bonneh et al (2001) found that the arrangement of stationary elements disappeared more often as a whole when it made up a 'good Gestalt', defined by proximity or contour smoothness, indicating that MIB is susceptible to effects of perceptual grouping. In an MIB study by Mitroff and Scholl (2005), grouping effects in the reappearance of elements that have perceptually disappeared were investigated. First, participants had to indicate when two Gabor patches 
had perceptually disappeared. As soon as this was the case both elements were physically rotated, for instance until they were collinear with each other. The stimulus was thus modified while the subject was unaware of its presence. It was shown that the percentage of mutual reappearance in MIB is positively influenced by the effects of grouping. In a paradigm quite similar to the flash-induced fading paradigm, Moradi and Shimojo (2004) found grouping effects of orientation. In one of their experiments, two low-contrast Gabor patches were presented until adaptation occurred. The patches could be either collinear with or orthogonal to each other. Subsequently, after $8 \mathrm{~s}$ of adaptation, a high-contrast Gabor patch was briefly displayed for $20 \mathrm{~ms}$. A significantly higher percentage of mutual disappearance was found in the collinear condition than in the orthogonal condition.

The impact of perceptual grouping on visual awareness has also been investigated in studies on binocular rivalry. It was shown that (suppression of) visual awareness can be influenced by interocular featural similarity (eg Kovács et al 1996; Lee and Blake 2004). In addition, van Lier and de Weert (2003) found that activation of a colour in one eye can cancel the suppression of same-colour patches in that eye or in the other eye, faster than that of other colours. This effect of interocular colour-specific activation in dichoptic suppression can be regarded as another demonstration that the contents of visual awareness are, at least partly, determined by aspects of perceptual grouping.

Indications for the involvement of higher-level processes in visual awareness can be found in the growing amount of literature on the role of recurrent processing in feature binding (de Kamps and van der Velde 2001; Murray et al 2004), as well as in visual awareness (eg Lamme and Roelfsema 2000; Pascual-Leone and Walsh 2001; Ro et al 2003; Tong 2003). An example of the way in which higher-level processes can influence effects of perceptual fading comes from Lou (1999), who showed that selective attention can facilitate Troxler fading. In his experiment, attended discs faded away from awareness significantly more often than discs that were not attended to. So, the perceptual disappearance in Troxler fading can at least partly be controlled by (top-down) attentional processes.

In the present study, we investigate whether the flash-induced fading effect (Kanai and Kamitani 2003) can be influenced by colour similarity and shape similarity of visual elements. Finding such grouping effects would further indicate that the different perceptual fading phenomena share common characteristics. If it turns out that local adaptation is not the sole determinant of any of the perceptual fading phenomena, then this would be an indication that a general account of perceptual fading should also incorporate more global perceptual processes.

\section{Experiment}

\subsection{Method}

2.1.1 Participants. Twenty-five healthy volunteers (aged 19-33 years, mean age 22.6 years), four males and twenty-one females, participated in the experiment. Volunteers were all students at the Radboud University Nijmegen and were all naive with respect to the experimental questions. All participants had normal or corrected-to-normal vision and received either course credit or a $5 €$ payment for their participation.

2.1.2 Stimuli and design. The experiment was developed with Presentation software (Neurobehavioral Systems, Albany, USA) and conducted on a Pentium-4 PC with a 21 inch CRT monitor, which was placed $160 \mathrm{~cm}$ in front of the participants. A chinrest was used to restrict head movements, and responses were given on a standard keyboard (QWERTY). 


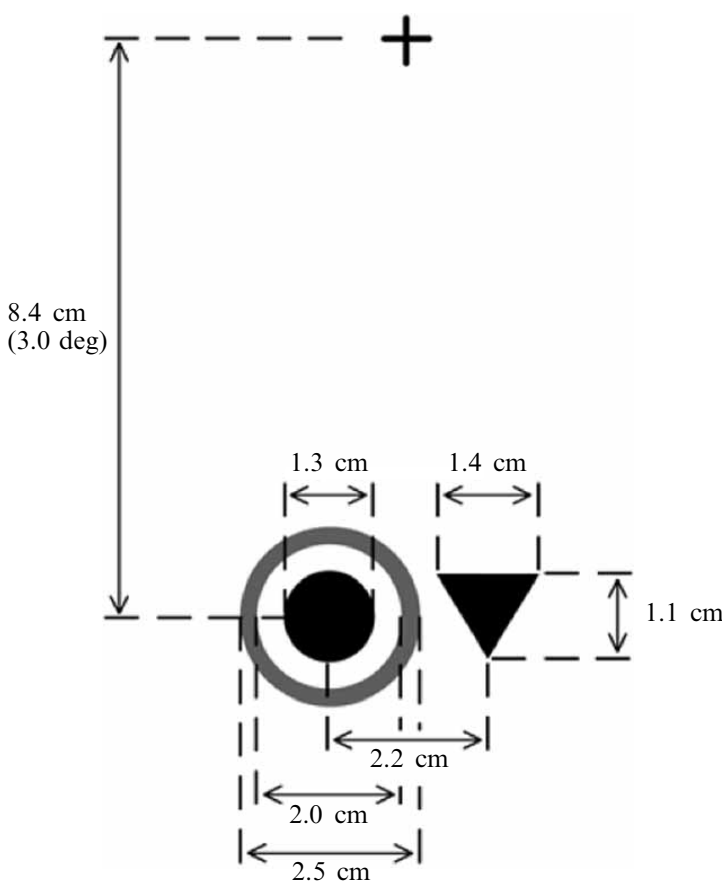

Figure 1. Configuration of the presentation of a flash display.

Stimuli were presented on a blue background [chromaticity value $\operatorname{CIE}(x, y)=0.20$, 0.17 ; luminance $L=7.65 \mathrm{~cd} \mathrm{~m}^{-2}$ ]. An example of a stimulus display with a flash (including distances between the different elements on the display) is given in figure 1.

Each stimulus consisted of either one or two elements, which were variable in both colour and shape. The elements were placed horizontally next to each other with a $2.2 \mathrm{~cm}(0.8 \mathrm{deg})$ distance between the element centres. The distance between the stimulus and a black fixation cross, which was presented above the stimulus, was $8.4 \mathrm{~cm}$ (3.0 deg). The shape of the elements could be circular, with a diameter of $1.3 \mathrm{~cm}$, or triangular, with a base of $1.4 \mathrm{~cm}$ and a height of $1.1 \mathrm{~cm}$, pointing downwards. The colour of the elements could be red $\left[\operatorname{CIE}(x, y)=0.57,0.34 ; L=17.79 \mathrm{~cd} \mathrm{~m}^{-2}\right.$ ] or green $\left[\operatorname{CIE}(x, y)=0.31,0.52 ; L=33.51 \mathrm{~cd} \mathrm{~m}^{-2}\right]$, with contrast values $\left(L_{\max }-L_{\min }\right) /$ $\left(L_{\max }+L_{\min }\right)$ of 0.40 and 0.63 , respectively. The white circular flash $\left(L=99.99 \mathrm{~cd} \mathrm{~m}^{-2}\right)$, which was presented around one of the two element locations, had an inner diameter of $2.0 \mathrm{~cm}$ and an outer diameter of $2.5 \mathrm{~cm}$. Monitor calibration and measurement of colour values and luminances were done with the aid of 'Colorshop 2.6/monitor optimizer', X-Rite, Inc.

2.1.3 Procedure. Each trial started with a $2000 \mathrm{~ms}$ period during which only the fixation cross was shown (see figure 2). Subsequently, the stimulus was presented (below the fixation cross) for $3000 \mathrm{~ms}$, after which a flash appeared around one of the element locations for $40 \mathrm{~ms}$. After disappearance of the flash, the stimulus remained on the screen for $2500 \mathrm{~ms}$ (which will hereafter be referred to as the critical period). Next, the screen was cleared, and at the same time a sound indicated that a response could be given.

Participants were instructed to indicate which element(s) had temporarily disappeared from awareness during the critical period of a trial. Four responses were possible: (1) only the left element had perceptually disappeared, (2) only the right element had perceptually disappeared, (3) both elements had perceptually disappeared, and (4) none of the elements had perceptually disappeared at any moment during the critical period. In trials in which one element was presented, only two responses were possible: (1) the element had perceptually disappeared during the critical period, 


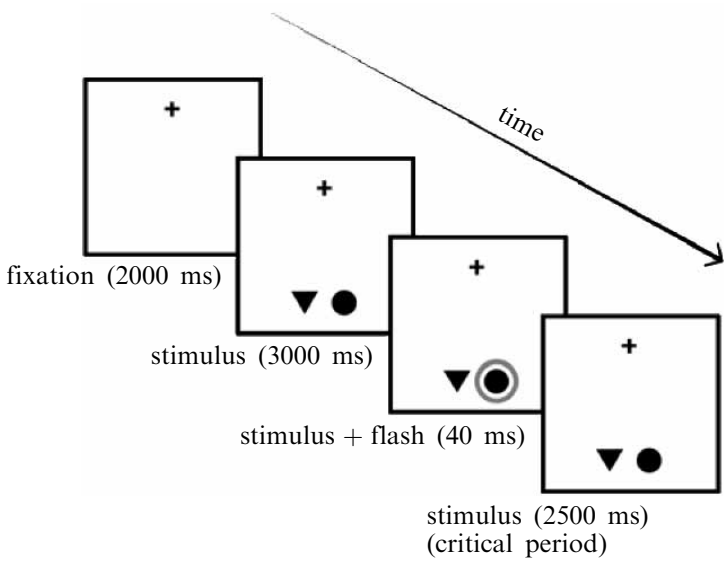

Figure 2. Schematic presentation of a trial.

and (2) the element was visible during the whole critical period. Responses were given with NUM-keys ' 1 ', '3', '+', and '-'.

When a trial consisted of two elements, these were either the same or different with respect to colour and/or shape (see figure 3). The design was balanced with respect to the number of trials in each colour and shape condition. The position of the stimulus elements and of the flash was counterbalanced across experimental trials.

The experiment consisted of a total of 192 experimental trials, preceded by 12 practice trials. The conditions in which two stimulus elements were presented contained 128 trials [colour similarity $(2) \times$ shape similarity $(2) \times$ flash side $(2) \times$ left $/$ right balance colours $(2) \times$ left/right balance shapes $(2) \times 4$ repetitions]. The single-element condition consisted of 64 trials [colour $(2) \times$ shape $(2) \times$ stimulus side $(2) \times$ flash side $(2) \times 4$ repetitions]. The experimental session contained three short breaks. The trials appeared in a randomised order and the total duration of the experiment was approximately $45 \mathrm{~min}$.

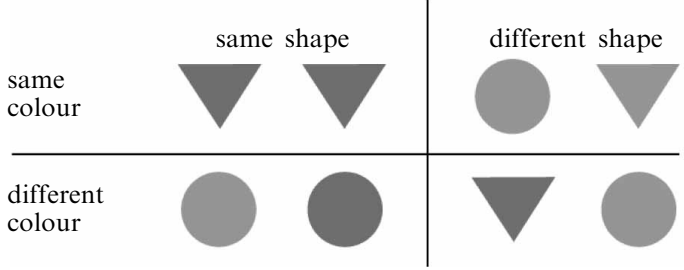

Figure 3. Examples of stimuli for both levels of the variables: colour and shape.

\section{Results}

For both the one-element and the two-elements conditions, we calculated the percentage of trials in which the element inside and the element outside the flash perceptually disappeared (figure 4). In the one-element condition, there was a higher percentage of perceptual disappearance when the element was inside the flash $(68.6 \%)$ than when the element was outside the flash $(29.0 \%)\left(t_{24}=5.374, p<0.001\right)$. Similarly, in the two-elements condition a significantly higher percentage of perceptual disappearance was observed for the element inside the flash $(64.7 \%)$ compared to the element outside the flash $(19.1 \%)\left(t_{24}=7.434, p<0.001\right)$. Overall, there was no difference in the number of disappearances between the two different colours (red versus green) or the two different shapes (discs versus triangles) used in the experiment $\left(t_{24}=1.105\right.$, $p=0.280$ and $t_{24}=0.743, p=0.465$, respectively).

Now we will focus on the trials in which two elements were presented. In $69.6 \%$ of these trials at least one of the elements perceptually disappeared; in the other $30.4 \%$ of the trials none of the elements perceptually disappeared. First we take a closer look at the latter trials. Paired-samples $t$-tests revealed that the percentage of 


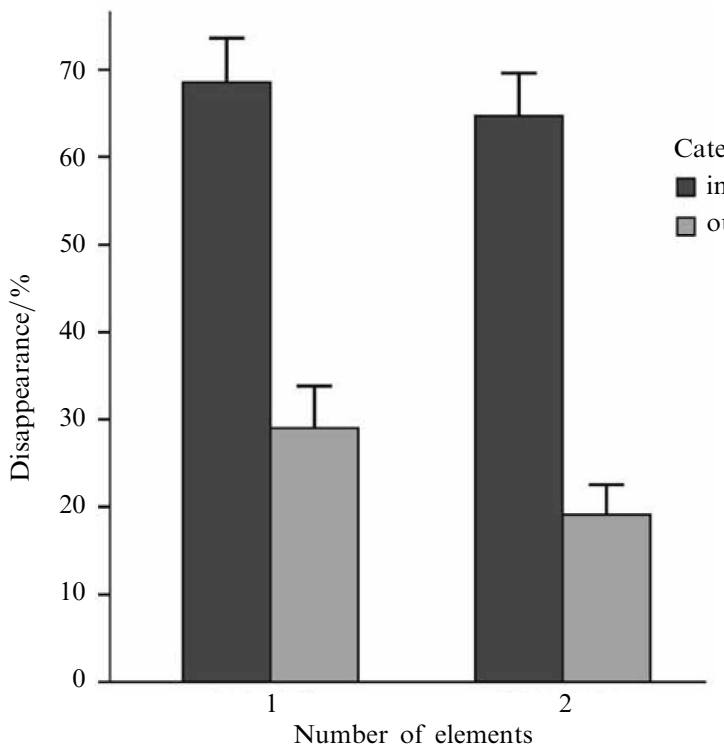

\author{
Category \\ $\square$ inside flash \\ outside flash
}

Figure 4. Percentage of disappearance of the elements presented inside the flash, as well as of those presented outside the flash. Results are presented for both the oneelement and the two-elements conditions. Error bars represent \pm 1 SEM.

trials in which none of the elements disappeared did not differ significantly between the same-colour and the different-colour conditions $\left(t_{24}=-0.786, p=0.440\right)$. This also holds for the same-shape and the different-shape conditions $\left(t_{24}=-0.553, p=0.585\right)$. Next, we consider the trials in which one or two elements perceptually disappeared. Obviously, the total number of these 'disappearance trials' equals the number of all trials minus the number of trials in which no element perceptually disappeared. For each participant the proportion of trials in which both elements had perceptually disappeared was calculated for each condition, given the disappearance of at least one element. This proportion will be referred to as PMD (proportion of mutual disappearance $): P M D=(\#[$ two elements disappeared $]) /(\#[$ trials $]-\#[$ no elements disappeared $])$. PMDs for each condition are presented in figure 5.

When the two elements were of the same colour, PMD was 0.234 (SE =0.043), and, when they were of a different colour, PMD was $0.187(\mathrm{SE}=0.039)$. Paired-samples $t$-tests showed that this effect of colour similarity was significant $\left(t_{24}=2.184, p<0.05\right)$. When the elements were of the same shape, PMD was $0.236(\mathrm{SE}=0.044)$, and, when they were of a different shape, PMD was 0.190 ( $\mathrm{SE}=0.038)$. This effect of shape similarity was also significant $\left(t_{24}=2.636, p<0.05\right)$. The similarity effects did not differ for the two colours and the two shapes.

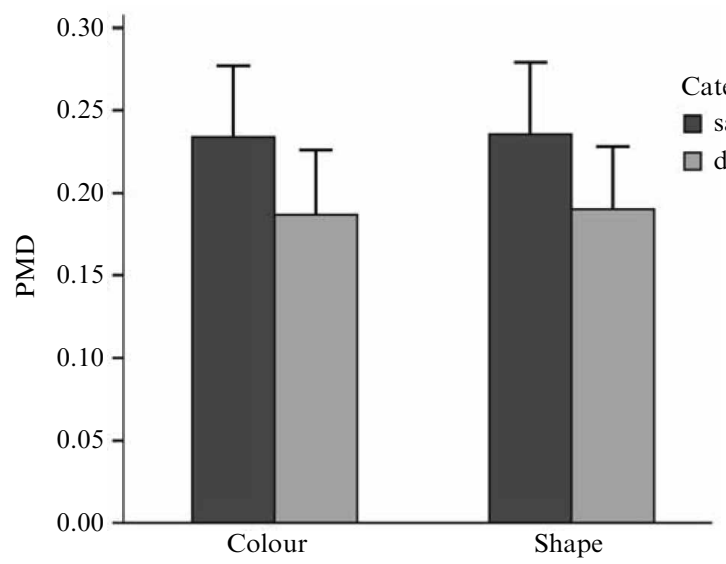

Figure 5. Proportions of mutual perceptual disappearance (PMDs) for the same and different colour category, as well as for the same and different shape category. Error bars represent \pm 1 SEM. 
In addition, the data show an additive trend: PMD was significantly higher when the elements were similar on only one feature compared to when none of the features were similar $\left(t_{24}=2.451, p<0.05\right)$, and PMD was significantly higher when the elements were similar on both features compared to when they were similar on only one feature $\left(t_{24}=3.456, p<0.01\right)$.

\section{Discussion}

The results show that flash-induced fading is susceptible to effects of perceptual grouping due to colour similarity and shape similarity. There was a significantly higher proportion of mutual disappearance of the two elements when these elements were similar with respect to colour and shape, respectively. The effects of colour and of shape similarity are rather similar to each other and both contribute to the likelihood of mutual fading.

In the introduction we described a number of perceptual fading phenomena which are evoked by different experimental manipulations. Whereas Troxler fading simply occurs during static presentation, MIB and flash-induced fading are triggered by simultaneously presented information. For MIB and flash-induced fading it has been argued that the competition between neural representations of visual objects causes fading to occur (Bonneh et al 2001; Kanai and Kamitani 2003). That is, competition seems to trigger the fading of peripherally presented elements. This does not imply that, without competition, fading would not occur for these elements. The underlying mechanisms of the various fading phenomena might therefore not be so different. Whereas earlier studies showed grouping effects in other perceptual fading phenomena (Bonneh et al 2001; Mitroff and Scholl 2005; Moradi and Shimojo 2004), the present study shows that these effects also occur in flash-induced fading as a result of colour and shape similarity. As these findings rule out the possibility that local sensory adaptation is the only cause of the flash-induced fading effect, more global processes must also be taken into account in providing a full explanation of the different fading phenomena.

The effects of grouping on perceptual fading found in the present study fit in with the growing amount of neuropsychological literature in which the role of recurrent or feedback processing in the emergence of visual awareness is suggested (eg Lamme and Roelfsema 2000; Pascual-Leone and Walsh 2001; Ro et al 2003; Tong 2003). It is argued that feedback activity is necessary for visual awareness to emerge. The level of this (object based) feedback activity is possibly reduced during fading. This reduction in activation is at least partly due to adaptation and can be increased by concurrent activation. The difference in explanation between Troxler fading on the one hand, and MIB and flash-induced fading on the other hand, would then be that in Troxler fading adaptation is sufficient to explain the reduction of recurrent processing, whereas in the induced fading phenomena (MIB and flash-induced fading) this reduction is also facilitated by concurrent information.

The present study provides evidence that flash-induced fading is susceptible to effects of perceptual grouping. Thus, besides local adaptation, more global processes appear to play a role in the flash-induced fading effect as well. Therefore, these global processes should be accounted for in a general explanation of perceptual fading phenomena.

Acknowledgments. This research has been made possible by a grant from the Netherlands Organisation for Scientific Research (NWO). Part of this study was presented at the 28th European Conference on Visual Perception in A Coruña, Spain. We would like to thank Charles de Weert, Arno Koning, Liang Lou, and two anonymous reviewers for their useful comments on a previous version of the manuscript. 


\section{References}

Bonneh Y S, Cooperman A, Sagi D, 2001 "Motion-induced blindness in normal observers" Nature $411798-801$

Kamps M de, Velde F van der, 2001 "Using a recurrent network to bind form, color and position into a unified percept" Neurocomputing 38-40 $523-528$

Kanai R, Kamitani Y, 2003 “Time-locked perceptual fading induced by visual transients" Journal of Cognitive Neuroscience $15664-672$

Kovács I, Papathomas T, Yang M, Fehér Á, 1996 "When the brain changes its mind: interocular grouping during binocular rivalry" Proceedings of the National Academy of Sciences of the USA $9315508-15511$

Lamme V A F, Roelfsema P R, 2000 "The distinct modes of vision offered by feedforward and recurrent processing" Trends in Neuroscience $23571-579$

Lee S-H, Blake R, 2004 "A fresh look at interocular grouping during binocular rivalry" Vision Research $44983-991$

Lier R van, Weert C M M de, 2003 "Intra- and interocular color-specific activation during dichoptic suppression" Vision Research 431111 - 1116

Lou L, 1999 "Selective peripheral fading: evidence for inhibitory sensory effect of attention" Perception $28519-526$

Mitroff S R, Scholl B J, 2005 "Forming and updating object representations without awareness: evidence from motion-induced blindness" Vision Research 45961 -967

Moradi F, Shimojo S, 2004 "Suppressive effect of sustained low-contrast adaptation followed by transient high-contrast on peripheral target detection" Vision Research 44449 -460

Murray S O, Schrater P, Kersten D, 2004 "Perceptual grouping and the interactions between visual cortical areas" Neural Networks $17695-705$

Pascual-Leone A, Walsh V, 2001 "Fast backprojections from the motion to the primary visual area necessary for visual awareness" Science $292510-512$

Ramachandran V S, Gregory R L, 1991 "Perceptual filling in of artificially induced scotomas in human vision" Nature $350699-702$

Ro T, Breitmeyer B, Burton P, Singhal N S, Lane D, 2003 "Feedback contributions to visual awareness in human occipital cortex" Current Biology $111038-1041$

Tong F, 2003 "Primary visual cortex and visual awareness" Nature Neuroscience 4 219-229

Troxler D, 1804 "Über das Verschwinden gegebener Gegenstände innerhalb unseres Gesichtskreises", in Ophthalmologische Bibliothek volume 2, Eds K Himly, J A Schmidt (Jena: Fromann) pp 1-119 


\section{PERTEPTION}

VOLUME 362007

www.perceptionweb.com

Conditions of use. This article may be downloaded from the Perception website for personal research by members of subscribing organisations. Authors are entitled to distribute their own article (in printed form or by e-mail) to up to 50 people. This PDF may not be placed on any website (or other online distribution system) without permission of the publisher. 